International Journal of Quantum Information

Vol. 15, No. 8 (2017) 1799001 (9 pages)

(c) World Scientific Publishing Company

DOI: $10.1142 /$ S0219749917990015

\title{
Author Index Volume 15 (2017)
}

Abdalla, S., see Naeim, I. H.

15 (2017) 1750035

Abulkasim, H., Hamad, S., Khalifa, A. and El Bahnasy, K., Quantum

15 (2017) 1750023

Akbari-Kourbolagh, Y., Entanglement criteria for the three-qubit states

Ali, M., Freezing dynamics of genuine entanglement and loss of genuine nonlocality under collective dephasing

Allevi, A., Bina, M., Olivares, S. and Bondani, M., Homodyne-like detection scheme based on photon-number-resolving detectors

Allevi, A., Chesi, G. and Bondani, M., Generation of quasi-singlemode twin-beam states in the high-intensity domain

Alonso-Sanz, R., see Situ, H.

Apollaro, T. J. G., see Lorenzo, S.

Arthur, T. T., see Martin, T.

Arthur, T. T., Martin, T. and Fai, L. C., Quantum correlations and coherence dynamics in qutrit-qutrit systems under mixed classical environmental noises

Assad, S. M., Bradshaw, M. and Lam, P. K., Phase estimation of coherent states with a noiseless linear amplifier

Bae, J., see Chang, J.

Bagrov, V. G., Gitman, D. M., Levin, A. D. and Meireles, M. S., Entanglement of two-Electron spin states in a double quantum dot

Banaszek, K., see Chrostowski, A.

Bartalucci, S., see Curceanu, C.

Bassi, A., see Curceanu, C.

Batle, J., see Naeim, I. H.

Bazzi, M., see Curceanu, C.

Belogolovskii, M., see Zhitlukhina, E.

Ben-Aryeh, Y. and Mann, A., Separability and entanglement of two qubits density matrices using Lorentz transformations

Benatti, F., Floreanini, R., Olivares, S. and Sindici, E., Noisy effects in interferometric quantum gravity tests

15 (2017) 1750049

15 (2017) 1750022

$15(2017) 1740016$

15 (2017) 1740021

15 (2017) 1750034

15 (2017) 1750037

15 (2017) 1750015

15 (2017) 1750047

15 (2017) 1750009

15 (2017) 1750019

15 (2017) 1750006

15 (2017) 1740005

15 (2017) 1740004

15 (2017) 1740004

15 (2017) 1750035

15 (2017) 1740004

15 (2017) 1740011

15 (2017) 1750044

15 (2017) 1740014

Bendahane, M., El Atiki, M. and Kassou-Ou-Ali, A., Two-state magnetic field Aharonov-Bohm effect and the wave-particle duality in a Mach-Zehnder interferometer

15 (2017) 1750032

15 (2017) 1740009

15 (2017) 1750020 
Benzimoune, B., see Jebli, L.

15 (2017) 1750001

Bertolucci, S., see Curceanu, C.

15 (2017) 1740004

Bharath, K., see Sirsi, S.

15 (2017) 1750042

Bhullar, J., see Taneja, D.

15 (2017) 1750008

Bina, M., see Allevi, A.

15 (2017) 1740016

Bolonek-Lason, K., Examining the effect of quantum strategies on symmetric conflicting interest games

Bondani, M., see Allevi, A.

Bondani, M., see Allevi, A.

Bradshaw, M., see Assad, S. M.

Bragadireanu, M., see Curceanu, C.

Brambilla, E., see Gatti, A.

Cargnelli, M., see Curceanu, C.

Chang, J., Bae, J. and Kwon, Y., Distilling entanglement with noisy operations

Chen, J.-G., see Wang, M.-M.

Chesi, G., see Allevi, A.

Chrostowski, A., Demkowicz-Dobrzański, R., Jarzyna, M. and Banaszek, K., On super-resolution imaging as a multiparameter estimation problem

15 (2017) 1750033

15 (2017) 1740016

15 (2017) 1740021

15 (2017) 1750009

15 (2017) 1740004

15 (2017) 1740017

15 (2017) 1740004

15 (2017) 1750019

15 (2017) 1750012

15 (2017) 1740021

$15(2017) 1740005$

Ciaglia, F. M., Ibort, A. and Marmo, G., Geometrical structures for classical and quantum probability spaces

Cialdi, S., see Rossi, M. A. C.

Ciccarello, F., see Lorenzo, S.

Clozza, A., see Curceanu, C.

Cohen, E., see Paneru, D.

Collince, F. G., see Martin, T.

Croca, J. R., Quantum world return to a realistic view

Crosson, E., see Kong, L.

Cruz, C., Quantum correlations and Bell's inequality violation in a Heisenberg spin dimer via neutron scattering

Curceanu, C., Sirghi, D., Sirghi, F., Bartalucci, S., Bazzi, M.,

Clozza, A., De Paolis, L., Guaraldo, C., Iliescu, M., Miliucci, M., Pietreanu, D., Scordo, A., Shi, H., Sperandio, L., Bassi, A.,

Donadi, S., Milotti, E., Bertolucci, S., Bragadireanu, M.,

Cargnelli, M., Marton, J., Pichler, A., Zmeskal, J., Di Matteo, S., Egger, J. P., Laubenstein, M., Piscicchia, K. and Doce, O. V., Quantum mechanics under X-rays in the Gran Sasso underground laboratory

15 (2017) 1740004

15 (2017) 1740003

Daneshgaran, F., Mondin, M. and Olia, K., Quantization of high dimensional Gaussian vector using permutation modulation with application to information reconciliation in continuous variable QKD

15 (2017) 1740007

15 (2017) 1740009

15 (2017) 1740026

15 (2017) 1740004

15 (2017) 1740019

15 (2017) 1750015

15 (2017) 1740023

15 (2017) 1750011

15 (2017) 1750031

Daoud, M., see Jebli, L.

15 (2017) 1740028

15 (2017) 1750001

15 (2017) 1750020 
De Leo, N., see Zhitlukhina, E.

15 (2017) 1740011

De Martini, F., The quantum Higgs field and the resolution of the cosmological constant paradox in the Weyl-geometrical Universe Demkowicz-Dobrzański, R., see Chrostowski, A.

De Paolis, L., see Curceanu, C.

de Souza, G., see Nogueira, E. C.

de Vries, M., see Škorić, B.

Di Matteo, S., see Curceanu, C.

Doce, O. V., see Curceanu, C.

Dodonov, V. V., Uncertainty relations for two observables coupled with the third one

Donadi, S., see Curceanu, C.

Dousttalab, N., see Haghparast, M.

Duplinskiy, A., see Kanapin, A.

Egger, J. P., see Curceanu, C.

Eghbalifam, F., see Jafarizadeh, M. A.

El Atiki, M., see Bendahane, M.

El Bahnasy, K., see Abulkasim, $\mathrm{H}$.

Elze, H.-T., Ontological states and dynamics of discrete (pre-) quantum systems

Fai, L. C., see Martin, T.

Fai, L. C., see Kenfack, L. T.

Fai, L. C., see Arthur, T. T.

Farouk, A., see Naeim, I. H.

Filippov, S. N., Quantum dynamics intervened by repeated nonselective measurements

Floreanini, R., see Benatti, F.

Fouokeng, G. C., see Kenfack, L. T.

Fretto, M., see Zhitlukhina, E.

Gatti, A. and Brambilla, E., Continuous variable entanglement of counter-propagating twin beams

Gheibi, R., see Heidari, S.

Giraldi, F., Bath correlation functions for logarithmic-like spectral densities

15 (2017) 1740025

15 (2017) 1740005

15 (2017) 1740004

15 (2017) 1750045

15 (2017) 1750016

15 (2017) 1740004

15 (2017) 1740004

15 (2017) 1740015

15 (2017) 1740004

15 (2017) 1750024

15 (2017) 1740018

15 (2017) 1740004

15 (2017) 1750055

15 (2017) 1750032

15 (2017) 1750023

15 (2017) 1740013

15 (2017) 1750015

15 (2017) 1750038

15 (2017) 1750047

15 (2017) 1750035

15 (2017) 1740027

15 (2017) 1740014

15 (2017) 1750038

15 (2017) 1740011

15 (2017) 1740017

15 (2017) 1750039

15 (2017) 1740022

Giraldi, F., Sequences of information backflow in local dephasing channels with spectral gaps

15 (2017) 1750050

15 (2017) 1750006

15 (2017) 1740004

15 (2017) 1750052

15 (2017) 1750017

15 (2017) 1750008

Gupta, M., see Taneja, D.

Haghparast, M. and Dousttalab, N., Design of new reversible quaternary flip-flops

15 (2017) 1750024

15 (2017) 1750023

Hamad, S., see Abulkasim, H.

Hansen, L. O. and Myrheim, J., Nongeneric positive partial transpose states of rank five in $3 \times 3$ dimensions

15 (2017) 1750054 
Hao, X., see $\mathrm{Wu}, \mathrm{W} .-\mathrm{J}$.

15 (2017) 1750027

He, G. P., Quantum private comparison protocol without a third party

15 (2017) 1750014

He, K., Wei, D. and Wang, L., Sum uncertainty relations for mixed

15 (2017) 1750030

He, Y.-F. and Ma, W.-P., Two-party quantum key agreement with five-particle entangled states

$15(2017) 1750018$

Heidari, S., Pourarian, M. R., Gheibi, R., Naseri, M. and Houshmand, M., Quantum red-green-blue image steganography

Holweck, F. and Saniga, M., Contextuality with a small number of observables

Hou, J., see Wang, Y.

Houshmand, M., see Nanvakenari, M.

Houshmand, M., see Heidari, S.

Hradil, Z., see Teo, Y. S.

Huang, Q., see Zhang, C.

Ibort, A., see Ciaglia, F. M.

Iliescu, M., see Curceanu, C.

Iliyasu, A. M., see Yan, F.

Jafarizadeh, M. A., Eghbalifam, F., Nami, S. and Yahyavi, M., Entanglement classification in the noninteracting Fermi gas

Jarzyna, M., see Chrostowski, A.

Jebli, L., Benzimoun, B. and Daoud, M., Quantum correlations for two-qubit $X$ states through the local quantum uncertainty

Jebli, L., Benzimoune, B. and Daoud, M., Local quantum uncertainty for a class of two-qubit $X$ states and quantum correlations dynamics under decoherence

Jeong, H., see Teo, Y. S.

Jin, M.-L., see Liu, F.-M.

Joyia, W. and Khan, K., Dzyaloshinskii-Moriya interaction and anisotropy effects on the tripartite quantum discord of Heisenberg $X Y$ model

15 (2017) 1750039

15 (2017) 1750026

15 (2017) 1750041

15 (2017) 1750002

15 (2017) 1750039

15 (2017) 1740002

15 (2017) 1750010

15 (2017) 1740007

15 (2017) 1740004

15 (2017) 1730001

15 (2017) 1750055

15 (2017) 1740005

15 (2017) 1750020

15 (2017) 1750001

15 (2017) 1740002

15 (2017) 1750036

15 (2017) 1750021

Kanapin, A., Duplinskiy, A., Sokolov, A., Vorobey, S., Miller, A., Kurochkin, V. and Kurochkin, Y., Urban QKD test for phase and polarization encoding devices

Kassou-Ou-Ali, A., see Bendahane, M.

Kenfack, L. T., Tchoffo, M., Fouokeng, G. C. and Fai, L. C., Dynamics of tripartite quantum correlations in mixed classical environments: The joint effects of the random telegraph and static noises

15 (2017) 1750038

15 (2017) 1750023

15 (2017) 1750051

15 (2017) 1750021

Khan, K., see Joyia, W.

15 (2017) 1740018

15 (2017) 1750032

Khrennikov, A., Buonomano against Bell: Nonergodicity or nonlocality?

15 (2017) 1740010 
Kitaeva, G. Kh. and Kornienko, V. V., Strongly nondegenerate spontaneous parametric down-conversion for calibration of terahertz-wave detectors

15 (2017) 1740024

Kong, L. and Crosson, E., The performance of the quantum adiabatic algorithm on spike Hamiltonians

15 (2017) 1750011

Kornienko, V. V., see Kitaeva, G. Kh.

15 (2017) 1740024

Koroli, V. I., Palistrant, S. and Nistreanu, A., Higher-order squeezing oscillations of the single-mode cavity field interacting with a three-level radiator

15 (2017) 1740012

15 (2017) 1740018

15 (2017) 1740018

15 (2017) 1750019

15 (2017) 1750009

15 (2017) 1740004

15 (2017) 1730001

15 (2017) 1750006

15 (2017) 1750034

15 (2017) 1750017

15 (2017) 1750052

Li, R., see Liu, Y.

Li, S.-S., Squeezing and entanglement in two-mode Bose-Einstein condensates

Lin, J.-Z., Scheme for quantum state manipulation in coupled cavities

15 (2017) 1750046

15 (2017) 1750004

Liu, F.-M. and Jin, M.-L., Research on information quantization based on the quantum theory

Liu, F., Quantum mutual information matrices

Liu, Y., Li, R., Lü, L. and Guo, L., New quantum codes derived from a family of antiprimitive $\mathrm{BCH}$ codes

Liu, Z., see Qiu, L.

15 (2017) 1750036

15 (2017) 1750005

15 (2017) 1750052

15 (2017) 1750025

Lorenzo, S., Apollaro, T. J. G., Trombettoni, A. and Paganelli, S., 2-qubit quantum state transfer in spin chains and cold atoms with weak links

15 (2017) 1750037

Lorenzo, S., Ciccarello, F. and Palma, G. M., Non-Markovian dynamics from band edge effects and static disorder

Lü, L., see Liu, Y.

Lu, L., Li, R. and Guo, L., Entanglement-assisted quantum codes from quaternary codes of dimension five

Ma, W.-P., see He, Y.-F.

Mancini, S., see Rexiti, M.

Mann, A., see Ben-Aryeh, Y.

Marmo, G., see Ciaglia, F. M.

Martin, T., see Arthur, T. T.

15 (2017) 1740026

15 (2017) 1750052

15 (2017) 1750017

15 (2017) 1750018

15 (2017) 1750053

15 (2017) 1750044

15 (2017) 1740007

15 (2017) 1750047

Martin, T., Arthur, T. T., Collince, F. G. and Fai, L. C., Time evolution of quantum correlations in superconducting flux-qubits under classical noises

15 (2017) 1750015

15 (2017) 1740004

Marton, J., see Curceanu, C.

15 (2017) 1750006 
Meis, C. and Dahoo, P. R., Vector potential quantization and the photon intrinsic electromagnetic properties: Towards nondestructive photon detection

Miliucci, M., see Curceanu, C.

Miller, A., see Kanapin, A.

Milotti, E., see Curceanu, C.

Mirafzali, S. Y., see Sargolzahi, I.

Mondin, M., see Daneshgaran, F.

Müller, C. R., see Teo, Y. S.

Myrheim, J., see Hansen, L. O.

Naeim, I. H., Batle, J., Abdalla, S. and Farouk, A., Nonlocality dynamics for an eight-qubit model in cavity QEDs

Nami, S., see Jafarizadeh, M. A.

Nanvakenari, M. and Houshmand, M., An efficient controlled quantum secure direct communication and authentication by using four particle cluster states

Narula, R., see Taneja, D.

Naseri, M., see Heidari, S.

$\mathrm{Ni}$, J., see Xiao, H.

Niestegge, G., Quantum key distribution without the wavefunction

Nikolić, H., Interpretation miniatures

Nistreanu, A., see Koroli, V. I.

Nogueira, E. C., de Souza, G., Varizi, A. D. and Sampaio, M. D., Quantum estimation in neutrino oscillations

Olia, K., see Daneshgaran, F.

Olivares, S., see Tamascelli, D.

Olivares, S., see Rossi, M. A. C.

Olivares, S., see Benatti, F.

Olivares, S., see Allevi, A.

Ouyang, S., see Xiao, H.

Paganelli, S., see Lorenzo, S.

Palistrant, S., see Koroli, V. I.

Palma, G. M., see Lorenzo, S.

Pan, F., see Qiu, L.

Paneru, D. and Cohen, E., Past of a particle in an entangled state

Paris, M. G. A., see Pozza, N. D.

Paris, M. G. A., see Rossi, M. A. C.

Parmar, J., Rahman, S. and Thiara, J., A formulation of a matrix sparsity approach for the quantum ordered search algorithm

Pastorello, D., A quantum key distribution scheme based on tripartite entanglement and violation of $\mathrm{CHSH}$ inequality

Patel, A. and Priyadarsini, A., Optimization of quantum Hamiltonian evolution: From two projection operators to local Hamiltonians

Pathak, A., see Thapliyal, K.

Pichler, A., see Curceanu, C.

Pietreanu, D., see Curceanu, C.

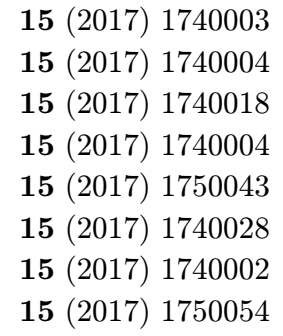

15 (2017) 1750035

15 (2017) 1750055

15 (2017) 1750002

15 (2017) 1750008

15 (2017) 1750039

15 (2017) 1750003

15 (2017) 1750048

15 (2017) 1740001

15 (2017) 1740012

15 (2017) 1750045

15 (2017) 1740028

15 (2017) 1740006

15 (2017) 1740009

15 (2017) 1740014

15 (2017) 1740016

15 (2017) 1750003

15 (2017) 1750037

15 (2017) 1740012

15 (2017) 1740026

15 (2017) 1750025

15 (2017) 1740019

15 (2017) 1750029

15 (2017) 1740009

15 (2017) 1750013

15 (2017) 1750040

15 (2017) 1650027

15 (2017) 1750007

15 (2017) 1740004

15 (2017) 1740004 
Piscicchia, K., see Curceanu, C.

15 (2017) 1740004

Pourarian, M. R., see Heidari, S.

15 (2017) 1750039

Pozza, N. D. and Paris, M. G. A., An effective iterative method to build the Naimark extension of rank-n POVMs

15 (2017) 1750029

15 (2017) 1650027

Prudêncio, T., Optical and spin-optical superpositions modulated by Aharonov-Bohm effect

Qi, X., see Wang, Y.

Qiu, L., Pan, F. and Liu, Z., Tripartite Bell-type inequalities for quantum coherence and skew information

$\mathrm{Qu}, \mathrm{Z} .-\mathrm{G}$. , see Wang, M.-M.

Raffa, F. A. and Rasetti, M., Full spectrum of nonlinear Jaynes-Cummings and anti-Jaynes-Cummings models

Rahman, S., see Parmar, J.

Rasetti, M., see Raffa, F. A.

Řeháček, J., see Teo, Y. S.

15 (2017) 1750028

15 (2017) 1750041

15 (2017) 1750025

15 (2017) 1750012

15 (2017) 1740008

15 (2017) 1750013

15 (2017) 1740008

15 (2017) 1740002

Rexiti, M. and Mancini, S., Estimation of two-qubit interactions through channels with environment assistance

15 (2017) 1750053

Rossi, M. A. C., Benedetti, C., Tamascelli, D., Cialdi, S., Olivares, S., Vacchini, B. and Paris, M. G. A., Non-Markovianity by undersampling in quantum optical simulators

Sampaio, M. D., see Nogueira, E. C.

Sánchez-Soto, L. L., see Teo, Y. S.

Saniga, M., see Holweck, F.

Sargolzahi, I. and Mirafzali, S. Y., Structure of states for which each localized dynamics reduces to a localized subdynamics

Scordo, A., see Curceanu, C.

Segati, A., see Tamascelli, D.

Seidel, P., see Zhitlukhina, E.

Sharma, R. D., see Thapliyal, K.

Shi, H., see Curceanu, C.

Shilpashree, S. P., see Sirsi, S.

Sindici, E., see Benatti, F.

Sirghi, D., see Curceanu, C.

Sirghi, F., see Curceanu, C.

Sirsi, S., Bharath, K., Shilpashree, S. P. and Smitha Rao, H. S., POVM construction: A simple recipe with applications to symmetric states

Situ, H., see Zhang, C.

Situ, H., Alonso-Sanz, R., Li, L. and Zhang, C., Land bidding game with conflicting interest and its quantum solution

15 (2017) 1740009

15 (2017) 1750045

15 (2017) 1740002

15 (2017) 1750026

15 (2017) 1750043

15 (2017) 1740004

15 (2017) 1740006

15 (2017) 1740011

15 (2017) 1750007

15 (2017) 1740004

15 (2017) 1750042

15 (2017) 1740014

15 (2017) 1740004

15 (2017) 1740004

15 (2017) 1750042

15 (2017) 1750010

$15(2017) 1750034$

Škorić, B. and de Vries, M., Quantum Key Recycling with 8-State encoding (The Quantum One-time Pad is more interesting than we thought)

15 (2017) 1750016

15 (2017) 1750042

15 (2017) 1740018

Smitha Rao, H. S., see Sirsi, S.

Sokolov, A., see Kanapin, A. 
Sosso, A., see Zhitlukhina, E.

Sperandio, L., see Curceanu, C.

Tamascelli, D., see Rossi, M. A. C.

Tamascelli, D., Segati, A. and Olivares, S., Dephasing assisted transport on a biomimetic ring structure

Tamma, V., see Zimmermann, O.

Taneja, D., Gupta, M., Narula, R. and Bhullar, J., Construction of new quantum MDS codes derived from constacyclic codes

Tchoffo, M., see Kenfack, L. T.

Teo, Y. S., Müller, C. R., Jeong, H., Hradil, Z., Řeháček, J. and Sánchez-Soto, L. L., Joint measurement of complementary observables in moment tomography

Thapliyal, K., Sharma, R. D. and Pathak, A., Protocols for quantum binary voting

Thiara, J., see Parmar, J.

Trombettoni, A., see Lorenzo, S.

Vacchini, B., see Rossi, M. A. C.

Varizi, A. D., see Nogueira, E. C.

Vorobey, S., see Kanapin, A.

Wang, L., see He, K.

Wang, M.-M., Qu, Z.-G., Wang, W. and Chen, J.-G., Effect of noise on joint remote preparation of multi-qubit state

Wang, W., see Wang, M.-M.

Wang, Y., Hou, J. and Qi, X., Quantum correlation based on weak measurements

Wei, D., see He, K.

Wu, W.-J., Yan, K., Xie, Y.-Q., Wu, Y. and Hao, X., Quantum speed-up dynamical crossover in open systems

$\mathrm{Wu}, \mathrm{Y} .$, see $\mathrm{Wu}, \mathrm{W} .-\mathrm{J}$.

Xiao, H., Ni, J., Xie, W. and Ouyang, S., A construction of quantum turbo product codes based on CSS-type quantum convolutional codes

Xie, W., see Xiao, H.

Xie, Y.-Q., see Wu, W.-J.

Yahyavi, M., see Jafarizadeh, M. A.

Yan, F., Iliyasu, A. M. and Le, P. Q., Quantum image processing: A review of advances in its security technologies

Yan, K., see Wu, W.-J.

Yang, P., see Zhang, C.

Zarei, M. H. and Khalili, Y., Systematic study of the completeness of two-dimensional classical $\phi^{4}$ theory

Zhang, C., see Situ, H.

Zhang, C., Situ, H., Huang, Q. and Yang, P., Multi-party quantum summation without a trusted third party based on single particles
15 (2017) 1740011

15 (2017) 1740004

15 (2017) 1740009

15 (2017) 1740006

15 (2017) 1740020

15 (2017) 1750008

15 (2017) 1750038

15 (2017) 1740002

15 (2017) 1750007

15 (2017) 1750013

15 (2017) 1750037

15 (2017) 1740009

15 (2017) 1750045

15 (2017) 1740018

15 (2017) 1750030

15 (2017) 1750012

15 (2017) 1750012

15 (2017) 1750041

15 (2017) 1750030

15 (2017) 1750027

15 (2017) 1750027

15 (2017) 1750003

15 (2017) 1750003

15 (2017) 1750027

15 (2017) 1750055

15 (2017) 1730001

15 (2017) 1750027

15 (2017) 1750010

15 (2017) 1750051

15 (2017) 1750034

15 (2017) 1750010 
Zhitlukhina, E., Belogolovskii, M., De Leo, N., Fretto, M., Sosso, A. and Seidel, P., Quantum coherent transport in a three-arm beam splitter and a Braess paradox

15 (2017) 1740011

Zimmermann, O. and Tamma, V., Which role does multiphoton interference play in small phase estimation in quantum Fourier transform interferometers?

15 (2017) 1740020

Zmeskal, J., see Curceanu, C.

15 (2017) 1740004 